\title{
Refractory hypoglycemia controlled by systemic chemotherapy with advanced hepatocellular carcinoma: A case report
}

\author{
JEN-SENG HUANG and PEI-HUNG CHANG \\ Division of Hemato-Oncology, Department of Internal Medicine, Chang Gung Memorial Hospital, Keelung, Taiwan, R.O.C.
}

Received November 20, 2014; Accepted October 5, 2015

DOI: $10.3892 / \mathrm{ol} .2015 .3915$

\begin{abstract}
Non-islet cell tumor hypoglycemia (NICTH) is an uncommon but serious complication of malignancy. Patients with NICTH may appear unwell due to the underlying tumor, particularly when the mechanism of hypoglycemia is extensive tumor burden in the liver. Hepatocellular carcinoma (HCC) is reported to be the second most common cause of NICTH. The therapeutic strategies used in treating NICTH involve reduction of the tumor mass or tumor load, and palliative treatment of symptoms if curative attempts fail. In the present study we report the successful control of hypoglycemia using systemic chemotherapy in an advanced HCC patient who presented with refractory NICTH.
\end{abstract}

\section{Introduction}

Patients with advanced hepatocellular carcinoma (HCC) occasionally develop a paraneoplastic syndrome that manifests as hypoglycemia, erythrocytosis, hypercalcemia or severe watery diarrhea, and is generally associated with a poor prognosis (1). Hypoglycemia, which normally occurs in advanced HCC, is understood to develop due to the tumor's high metabolic requirements. Hypoglycemia is typically mild; however, more severe reductions in blood sugar may occur, resulting in lethargy and confusion. Less than 5\% of tumors secrete insulin-like growth factor II (IGF-II), which results in stimulation of the insulin receptors and increased glucose utilization and could cause severe symptomatic hypoglycemia $(2,3)$. Hypoglycemia may be caused by several tumors, including islet and non-islet tumors. Non-islet cell tumor hypoglycemia (NICTH) is an uncommon but serious complication of malignancy (4). Systemic chemotherapy was previously demonstrated to lead to an unfavorable outcome for hypoglycemia in patients with HCC (3). We report herein the successful control of hypoglycemia with systemic chemotherapy in an advanced HCC

Correspondence to: Dr Pei-Hung Chang, Division of Hemato-Oncology, Department of Internal Medicine, Chang Gung Memorial Hospital, No. 200, Lane 208, Jijin 1st Road, Keelung 8862, Taiwan, R.O.C.

E-mail: ph555chang@cgmh.org.tw

Key words: hepatoma, non-islet tumor hypoglycemia, chemotherapy patient who presented with refractory NICTH. The patient provided written informed consent.

\section{Case report}

A 54-year-old male presented at Chang Gung Memorial Hospital, Keelung, Taiwan in April 2012 due to a 2-month history of constant pain in the upper right abdomen and a weight loss of $9 \mathrm{~kg}$ in the previous 2 months. A clinical survey revealed chronic hepatitis B and multiple hepatic masses infiltrating both hepatic lobes. Ultrasonography-guided biopsy of the liver mass revealed tumor cells consistent with moderately differentiated HCC. A computed tomography (CT) scan revealed no evidence of extrahepatic spread of the tumor. Due to extensive tumor infiltration the hepatoma was considered to be inoperable and liver transplantation was not indicated at that time. The patient was then treated twice with selective hepatic artery chemoembolization with doxorubicin. The patient refused further anti-cancer treatment and was lost to follow-up thereafter.

At the end of January 2013, the patient was readmitted to our hospital due to recurrent episodes of drowsiness, dizziness, confusion and sweating, which were particularly notable in the early morning and partially relieved after eating. A CT scan of the liver revealed massive liver enlargement and multilocular infiltration by hepatoma (Fig. 1). The patient was on no medications and had no underlying diabetes mellitus. Blood glucose was low (1.9 mmol/l) but administration of glucose reversed the symptoms, demonstrating that they were caused by hypoglycemia. Laboratory tests revealed elevated transaminase levels with AST 189 U/1 (normal, <35 U/l), ALT 34 U/1 (normal, <35 U/l), and hyperbilirubinemia with total bilirubin $1.9 \mathrm{mg} / \mathrm{dl}$ (normal, $<1.5 \mathrm{mg} / \mathrm{dl}$ ). However, frequent hypoglycemia episodes developed following admission. Further laboratory tests performed during the hypoglycemic episodes revealed suppressed insulin $(<1 \mu \mathrm{U} / \mathrm{ml})$ and $\mathrm{C}$-peptide $(0.15 \mathrm{ng} / \mathrm{ml})$ levels, indicating that the hypoglycemia was not caused by endogenously or exogenously raised insulin levels. IGF-I levels $(43.42 \mathrm{ng} / \mathrm{ml}$; normal, 81-225 ng/ml) were also suppressed. The IGF-II and 'big' IGF-II levels could not be measured at our institution. The patient experienced further recurrent hypoglycemia (four episodes of glucose levels lower than $3 \mathrm{mmol} / \mathrm{l}$ in 3 days) despite his monitored diet and a continuous intravenous infusion of $10 \%$ glucose water solution. Glucocorticoid treatment was started, but did not reduce the frequency or severity of hypoglycemic episodes. Glucagon caused a transient glycemic reaction with 


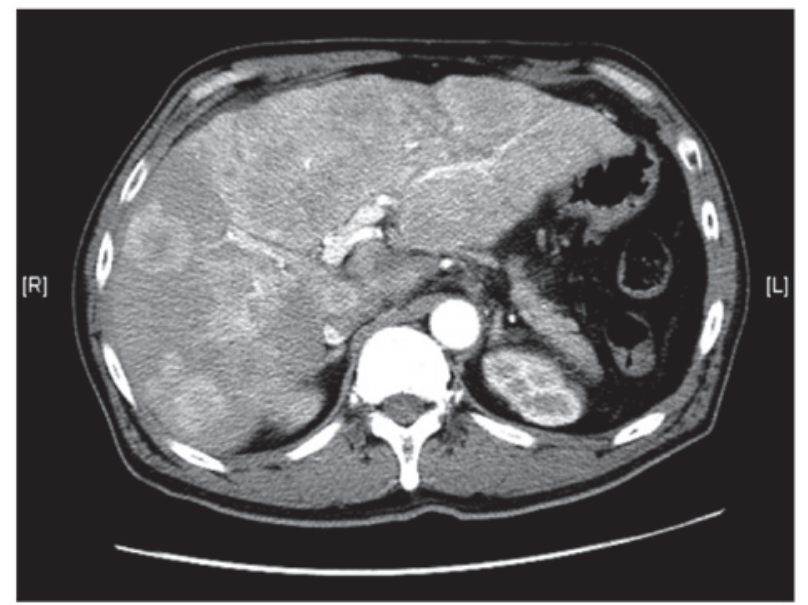

Figure 1. Computed tomography scan of the liver reveals massive liver enlargement and multilocular infiltration by hepatoma.

rapid relief of symptoms initially but the effect was not lasting. The frequency and severity of the hypoglycemic symptoms further progressed and refractory hypoglycemia was suspected due to NICTH caused by advanced hepatoma.

Therapeutic strategies for NICTH involve reduction of the tumor mass or tumor load and palliative treatment of symptoms if curative attempts fail. In consideration of the treatment benefit and administration safety, palliative systemic chemotherapy with oxaliplatin and 5-fluorouracil/leucovorin (FOLFOX4) were started; i.e., oxaliplatin $85 \mathrm{mg} / \mathrm{m}^{2}$ intravenously (IV) on day 1; LV $200 \mathrm{mg} / \mathrm{m}^{2}$ IV from hour 0 to 2 on days 1 and 2; and 5 -FU $400 \mathrm{mg} / \mathrm{m}^{2} \mathrm{IV}$ bolus at hour 2, then $600 \mathrm{mg} / \mathrm{m}^{2}$ over $22 \mathrm{~h}$ on days 1 and 2, once every 2 weeks. After two cycles of chemotherapy, the patient had not experienced any hypoglycemic attack, which allowed cessation of the corticoid and intravenous glucose. Grade 1 mucositis developed following chemotherapy, and was tolerated by the patient. The patient was then discharged from the hospital and maintained a good glycemic balance as an outpatient. However, chemotherapy was discontinued 3 months later due to further deterioration of the hepatic and pulmonary function with progression of the tumor. The patient succumbed to the disease at the end of June 2013.

\section{Discussion}

Severe hypoglycemia occurs in a small percentage of patients with non-islet cell tumors, usually of mesenchymal, vascular or epithelial cell types (4). NICTH is a rare but serious complication of the malignancy. HCC accounts for $23 \%$ of NICTH cases, making it the second-leading cause after mesenchymal tumors, which account for $45 \%$ of cases (5). Hypoglycemia in HCC is caused by impaired gluconeogenesis due to a decompensated liver (glucose underproduction) or by a high big IGF-II level produced by tumors, which results in stimulation of the insulin receptors and increased glucose utilization. In our patient, drug-induced hypoglycemia could be excluded, as the patient was not on any medication. Serum insulin and C-peptide levels were suppressed during hypoglycemia, indicating that the hypoglycemia was not caused by endogenously or exogenously raised insulin levels. In chronic liver disease and cirrhosis, the hepatic glucose metabolism is altered and hepatic glucose output in response to glucagon is decreased (6). However, at the onset of symptoms, our patient demonstrated no signs of advanced cirrhosis of the liver, hepatic function parameters were relatively normal, and the glycemic response to intravenous glucagon was adequate. Therefore, the hypoglycemia was not considered to be due to advanced cirrhosis. The diagnosis of NICTH is made when a patient demonstrates low insulin and $\mathrm{C}$-peptide levels together with an inappropriate increase in the ratio of IGF-II to IGF-I. Although measurements of the IGF-II and big IGF-II levels were not available for this patient, the low IGF-I, insulin and C-peptide levels noted during his attacks of hypoglycemia were suggestive of NICTH.

The treatment of NICTH involves immediate correction of hypoglycemia, treatment directed at the underlying malignancy and prevention of recurrent hypoglycemia in cases when it is not possible to control the tumor. Complete surgical resection remains the most effective therapeutic option (7); however, no effective therapy has emerged for advanced disease (8). Palliative treatment includes administration of counter-regulatory hormones including glucocorticoids, glucagon, growth hormone or octreotide $(3,9)$, although the effects may be transient. Percutaneous ethanol injection (10) or intrahepatic doxorubicin (11) administration may be beneficial in inoperable patients. Systemic chemotherapy was previously reported to be ineffective for hypoglycemia in patients with HCC (3), which contradicts the results demonstrated in the present case.

In our patient with refractory hypoglycemia caused by advanced hepatoma, the effectiveness of systemic chemotherapy in controlling hypoglycemia may have been partly due to its antitumoral activity. Sorafenib, at the forefront of therapy for advanced HCC, was not reimbursed in Taiwan at that time and not affordable for the patient. The efficacy of conventional cytotoxic chemotherapy is modest at best. In Asia, systemic chemotherapy with oxaliplatin and 5-fluorouracil/leucovorin (FOLFOX) is associated with a higher objective response rate (8\% vs. $3 \%$ ) and disease control rate (52\% vs. $32 \%)$ compared with single-agent doxorubicin (12). Despite the relatively shorter progression-free and overall survival time with this regimen (2.93 and 6.4 months, respectively), it was associated with modest antitumor activity in disease control and may have a benefit in the treatment of NICTH, as observed in our patient who made a good recovery from hypoglycemia. In conclusion, systemic chemotherapy with FOLFOX should be considered as a therapeutic option for NICTH in advanced HCC patients in whom tumor removal by surgery cannot be performed.

\section{Acknowledgements}

The authors thank all the members of the Cancer Center at Chang Gung Memorial Hospital for their assistance.

\section{References}

1. Luo JC, Hwang SJ, Wu JC, Lai CR, Li CP, Chang FY, Chiang JH, Lui WY, Chu CW and Lee SD: Clinical characteristics and prognosis of hepatocellular carcinoma patients with paraneoplastic syndromes. Hepatogastroenterology 49: 1315-1319, 2002.

2. Eastman RC, Carson RE, Orloff DG, Cochran CS, Perdue JF, Rechler MM, Lanau F, Roberts CT Jr, Shapiro J, Roth J, et al: Glucose utilization in a patient with hepatoma and hypoglycemia. Assessment by a positron emission tomography. J Clin Invest 89: 1958-1963, 1992. 
3. Tietge UJ, Schöfl C, Ocran KW, Wagner S, Böker KH, Brabant G, Zapf J and Manns MP: Hepatoma with severe non-islet cell tumor hypoglycemia. Am J Gastroenterol 93: 997-1000, 1998.

4. Scott K: Non-islet cell tumor hypoglycemia. J Pain Symptom Manage 37: e1-e3, 2009.

5. Kahn CR: The riddle of tumour hypoglycaemia revisited. Clin Endocrinol Metab 9: 335-360, 1980.

6. Petrides AS and DeFronzo RA: Glucose metabolism in cirrhosis: a review with some perspectives for the future. Diabetes Metab Rev 5: 691-709, 1989.

7. Kalebi AY, Hale MJ, Wong ML, Hoffman T and Murray J: Surgically cured hypoglycemia secondary to pleural solitary fibrous tumour: case report and update review on the Doege-Potter syndrome. J Cardiothorac Surg 4: 45, 2009.

8. Park MS and Araujo DM: New insights into the hemangiopericytoma/solitary fibrous tumor spectrum of tumors. Curr Opin Oncol 21: 327-331, 2009.

9. Perros P, Simpson J, Innes JA, Teale JD and McKnight JA: Non-islet cell tumour-associated hypoglycaemia: 111In-octreotide imaging and efficacy of octreotide, growth hormone and glucocorticosteroids. Clin Endocrinol (Oxf) 44: 727-731, 1996.
10. Saigal S, Nandeesh HP, Malhotra V and Sarin SK: A case of hepatocellular carcinoma associated with troublesome hypoglycemia: management by cytoreduction using percutaneous ethanol injection. Am J Gastroenterol 93: 1380-1381, 1998.

11. Hunter SJ, Daughaday WH, Callender ME, McKnight JA, McIlrath EM, Teale JD and Atkinson AB: A case of hepatoma associated with hypoglycaemia and overproduction of IGF-II (E-21): beneficial effects of treatment with growth hormone and intrahepatic adriamycin. Clin Endocrinol (Oxf) 41: 397-401, 1994; discussion 402.

12. Qin S, Bai Y, Lim HY, Thongprasert S, Chao Y, Fan J, Yang TS, Bhudhisawasdi V, Kang WK, Zhou Y, et al: Randomized, multicenter, open-label study of oxaliplatin plus fluorouracil/leucovorin versus doxorubicin as palliative chemotherapy in patients with advanced hepatocellular carcinoma from Asia. J Clin Oncol 31: 3501-3508, 2013 